\title{
Polyhedral Micromotors of Metal-Organic Frameworks: Symmetry Breaking and Propulsion
}

Zhisheng Wang, Wei Xu, Zuochen Wang, Dengping Lyu, Yijiang Mu, Wendi Duan, and

$$
\text { Yufeng Wang* }
$$

Department of Chemistry, The University of Hong Kong, Pokfulam Road, Hong Kong, 999077, China.

*To whom correspondence should be addressed: wanglab@hku.hk. 


\section{Materials and Methods}

\section{General methods}

All chemicals were purchased from commercial suppliers and used without further purification. Scanning electron microscope (SEM) images of metal-organic framework (MOF) particles were obtained with MAIA3 SEM; The samples were sputter-coated with a thin layer of gold for better imaging. Nikon Eclipse Ti-2 inverted microscope equipped with a N7000 DSLR camera were used to observe the particles. Trackpy package in Python was used to track the locomotive behaviors of particles and extract data for analysis.

\section{Synthesis of MIL-96 particles}

MIL-96 particles with different truncations were synthesized as the following based on the previous literature with modifications ${ }^{1}: 800 \mathrm{mg}$ of aluminum nitrate nonahydrate was dissolved into $4 \mathrm{~mL}$ of 1:3 dimethylformamide (DMF) /water solution in a 25-mL autoclave, to which $400 \mathrm{mg}$ of benzene-1,3,5-tricarboxylic acid in $4 \mathrm{~mL}$ of 1:3 DMF/water solution was added. Then up to $3 \mathrm{~mL}$ of acetic acid and $200 \mathrm{uL}$ of DMF was added to the autoclave so the total volume of reaction was about $8.5 \mathrm{~mL}$. The autoclave was sealed and put into an oven at 130 $210 \mathrm{C}^{\circ}$ for $24 \mathrm{hr}$. After cooling down, the particles were transferred into a $20-\mathrm{mL}$ vial and preserved in the mother liquor, which were washed by DMF and water before using. The amount of acetic acid and the reaction temperatures were adjusted to obtain MIL-96 particles of the THBP shape with different truncations.

\section{Synthesis of UiO-66, ZIF-8, MIL-88B particles}

UiO-66, ZIF-8, MIL-88B particles were synthesized following previous literature with some modifications $\mathrm{s}^{2-4}$. For UiO-66, $150 \mathrm{~mL}$ of DMF was used to dissolve $1.58 \mathrm{~g}$ zirconyl chloride octahydrate in a 500-mL Pyrex bottle, followed by adding $200 \mathrm{~mL}$ of acetic acid. Then, $3.75 \mathrm{~g}$ of terephthalic acid in $150 \mathrm{~mL}$ of DMF was added, and the mixture was incubated at room temperature for 1 hour and then sonicated for 20 mins. After 18 -hours heating at $90 \mathrm{C}^{\circ}$ 
and cooling down to room temperature, the product was washed by centrifugation/redispersion cycles using DMF for three times. For cubic ZIF-8, $20 \mathrm{ml}$ of water containing $0.29 \mathrm{~g}$ of zinc nitrate hexahydrate was added into $140 \mathrm{~mL}$ of water containing $4.54 \mathrm{~g}$ of 2-methylimidazole and $0.006 \mathrm{~g}$ of cetyltrimethylammonium bromide (CTAB), stirred for 5 mins. Then the mixture was incubated at room temperature for 6 hours and the products were collected by centrifugation. For rhombic dodecahedral ZIF-8, $1 \mathrm{ml}$ of $0.09 \mathrm{M}$ zinc acetate dihydrate aqueous solution was mixed with $1 \mathrm{~mL}$ of $1.15 \mathrm{M}$ 2-methylimidazole aqueous solution, vigorously shaken for 10s. Then the solution was incubated at room temperature for 7 hours and the particles were washed by centrifugation/redispersion cycles for three times. For MIL-88B, 49.7 $\mathrm{mg}$ of iron (III) chloride hexahydrate, $20.0 \mathrm{mg}$ of 1,4-benzendicarboxylic acid and $2.0 \mathrm{~mL}$ of $50 \mathrm{mM}$ Polyvinylpyrrolidone (PVP) 360k in DMF were mixed with $1.2 \mathrm{~mL}$ of DMF in a glass vial and homogenized by sonication. The solution was heated at $145{ }^{\circ} \mathrm{C}$ for 1.5 hours with stirring. After cooling down to room temperature, the particles were purified with DMF twice via centrifugation/redispersion cycles.

\section{AC electric field setup for MOF particles propulsion}

The electric cell was made by combining two iridium coated ITO glass, separated by the 50 - $\mu \mathrm{m}$-thick insulating film, leaving an empty cell. Copper wires were stick to the ITO glass as electrodes. MOF particles of interest were dispersed in deionized water (dilute sample, approximately $0.01 \mathrm{mg} / \mathrm{mL}$ ) containing a certain amount of ammonia and then charged into the cell. Square waves of electric field vertical to the glass, parameterized by voltages $\left(V_{\mathrm{pp}}\right)$ and frequencies $(f)$, were introduced by a function generator (RIGOL, DG1022U).

\section{Zeta potential measurement}

MOF particles were dispersed in deionized water (dilute sample, approximately 0.01 $\mathrm{mg} / \mathrm{mL}$ ) and then a certain amount ammonia or other reagents (see Table S3) were added, followed by sonicating the solution for 3 mins. The zeta potential measurements were carried out using Zetasizer Nano-ZS90 (Malvern). 


\section{Numerical Simulation with COMSOL Multiphysics}

We simulated the flow and distorted electric field around an MOF particle using COMSOL Multiphysics (Version 5.4a) software. We considered a 2D cross-section of the MOF particle (with a low dielectric constant) and an electric field setup at low frequencies $(f<2000 \mathrm{~Hz})$. A thin-double-layer approximation was accepted to calculate the electric field distribution, which mainly followed the methods by Ristenpart et $a l^{5}$.

$$
\nabla \varphi \cdot \mathbf{n}=-\lambda \nabla_{\mathrm{s}}^{2} \varphi
$$

where $\lambda$ is the dimensionless complex surface conductivity.

Afterwards, electroosmotic velocity was set as the boundary condition of the lower electrode. 


\section{Supplementary Figures and Tables}

Table S1. Geometry parameters of UiO-66, ZIF-8, MIL-88B particles.

\begin{tabular}{|c|c|}
\hline Type of MOF Particle & Edge/ $\boldsymbol{\mu m}$ \\
\hline UiO-66 & $1.76 \pm 0.02$ \\
\hline cubic ZIF-8 & $1.30 \pm 0.02$ \\
\hline rhombic dodecahedral ZIF-8 & $1.14 \pm 0.04$ \\
\hline MIL-88B & $2.02 \pm 0.07$ (side edge) \\
& $1.36 \pm 0.03$ (equatorial edge) \\
\hline
\end{tabular}
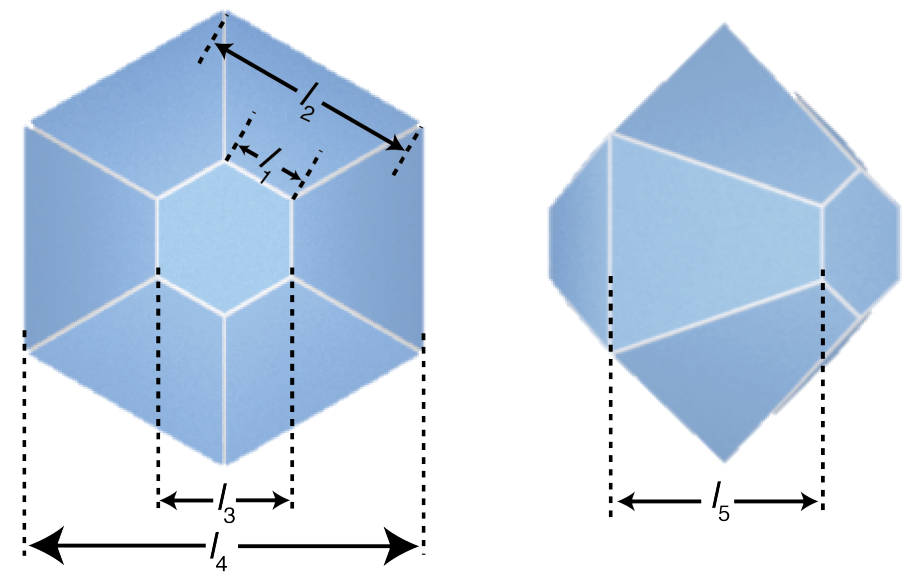

Figure S1. Scheme of geometry parameters of the MIL-96 particle (top view)

Table S2. Geometry parameter of MIL-96 particles with different truncations*

\begin{tabular}{|c|c|c|c|c|c|}
\hline MIL 96 particles & $\mathbf{1}$ & $\mathbf{2}$ & $\mathbf{3}$ & $\mathbf{4}$ & $\mathbf{5}$ \\
\hline$l_{3} / \mu \mathrm{m}$ & $0.67 \pm 0.03$ & $0.78 \pm 0.03$ & $0.89 \pm 0.03$ & $1.58 \pm 0.03$ & $1.40 \pm 0.25$ \\
\hline$l_{4} / \mu \mathrm{m}$ & $2.53 \pm 0.02$ & $2.70 \pm 0.04$ & $2.75 \pm 0.04$ & $3.35 \pm 0.04$ & $2.53 \pm 0.30$ \\
\hline$l_{5} / \mu \mathrm{m}$ & $1.38 \pm 0.02$ & $1.31 \pm 0.03$ & $1.33 \pm 0.03$ & $1.30 \pm 0.06$ & $0.85 \pm 0.04$ \\
\hline Dihedral angle/ & 132 & 137 & 134 & 133 & 132 \\
\hline Degree of truncation & 0.26 & 0.29 & 0.32 & 0.47 & 0.55 \\
\hline
\end{tabular}


*Degree of truncation $D_{\mathrm{tr}}=l_{1} / l_{2}$ or $l_{3} / l_{4}$

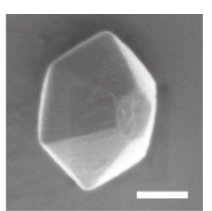

MIL-96-1

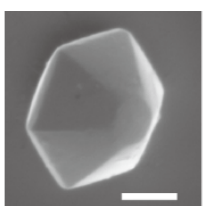

MIL-96-2

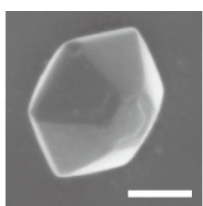

MIL-96-3

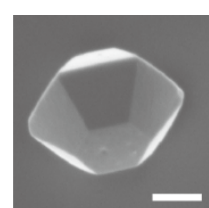

MIL-96-4

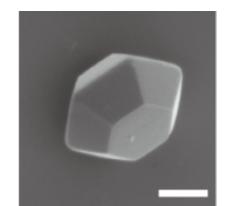

MIL-96-5

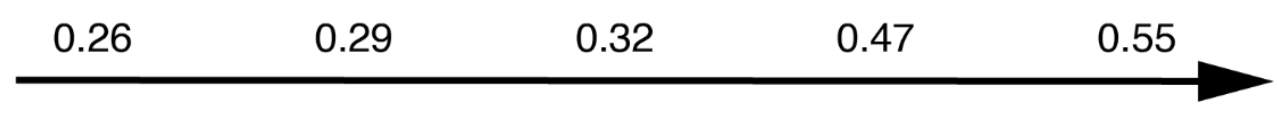

Increased degree of truncation

Figure S2. SEM images of MIL-96 particles with different truncations. Scale bar: $1 \mu \mathrm{m}$

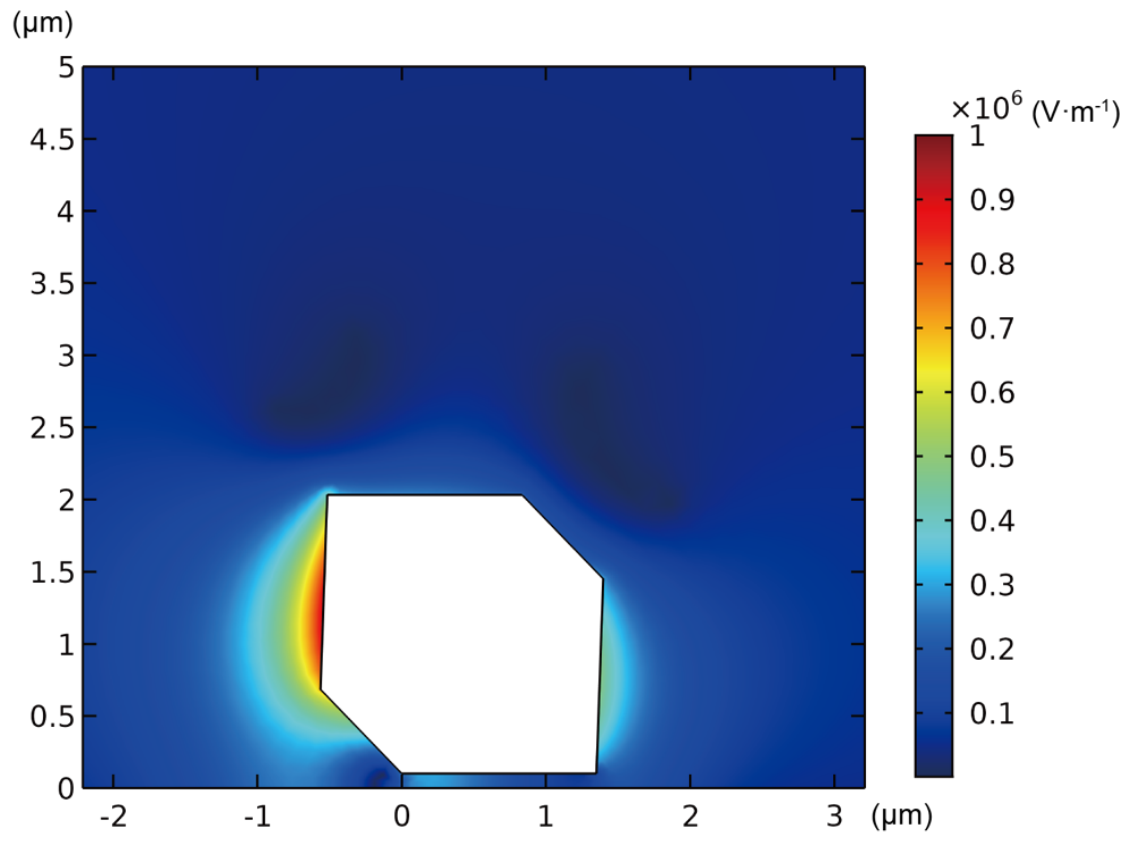

Figure S3. Infinite-element simulation of the nonuniform electric field around an MIL-96 particle (cross-section) by COMSOL Multiphysics. Color bar: strength of the electric fields. 

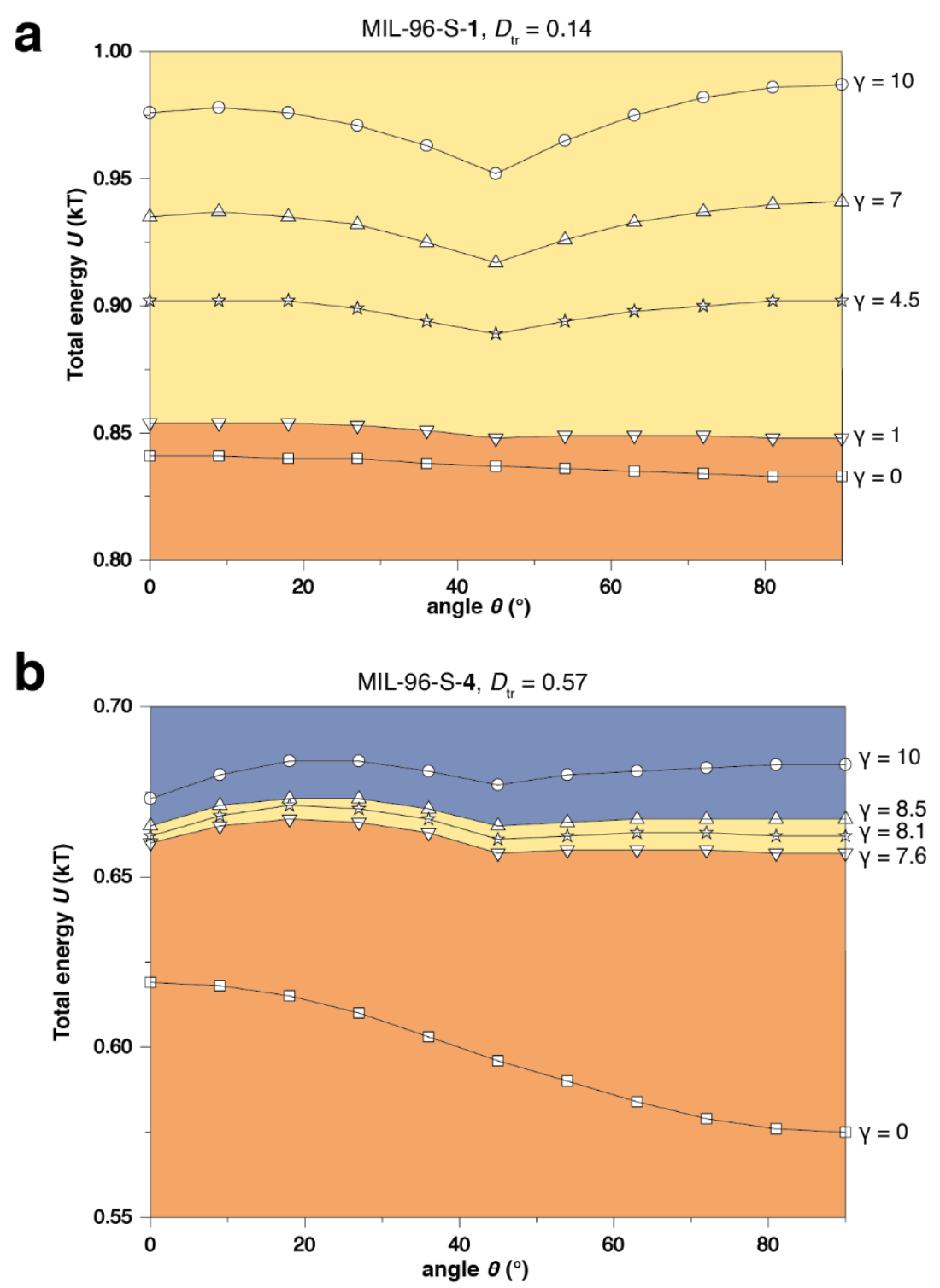

Figure S4. Theoretical modelling of the total energy $(U)$-orientation profile $(\theta)$ at different electric field strengths $(\gamma)$. MIL-96 particles with truncations of 0.14 (a) and 0.57 (b) are shown. 
a

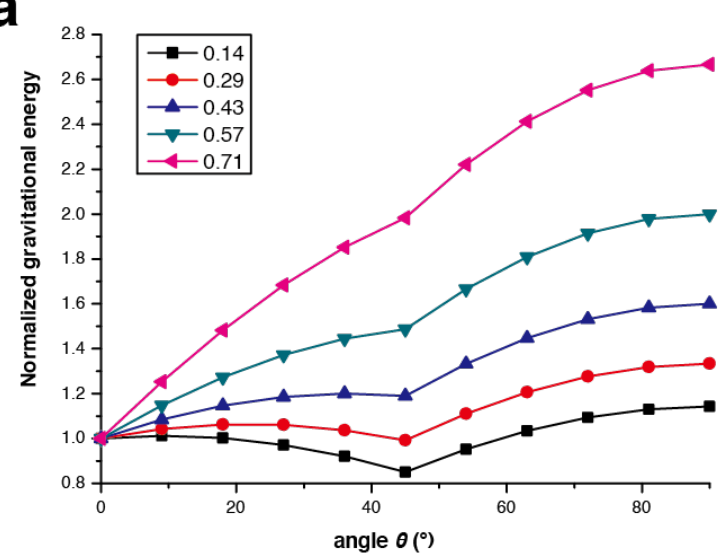

b

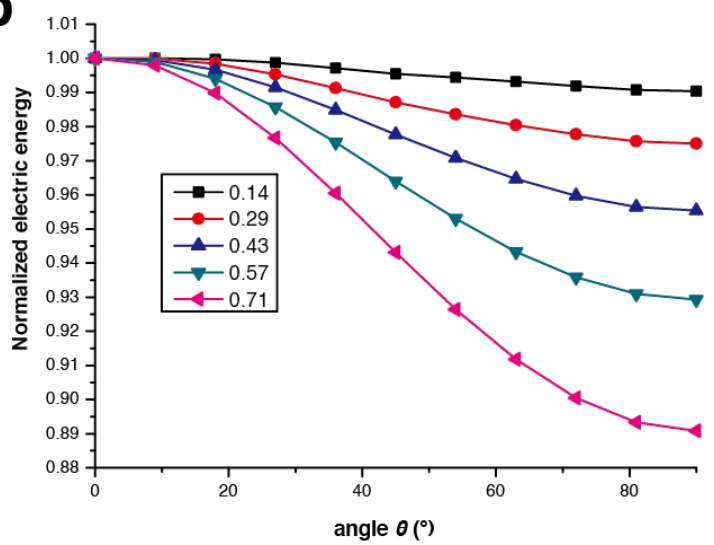

Figure S5. Theoretical energy profiles showing individual $U_{\mathrm{G}}(\mathrm{a})$ and $U_{\mathrm{E}}(\mathrm{b})$ of MIL-96 particles with different truncations. Each curve is normalized by the energy at angle $\theta=0^{\circ}$.

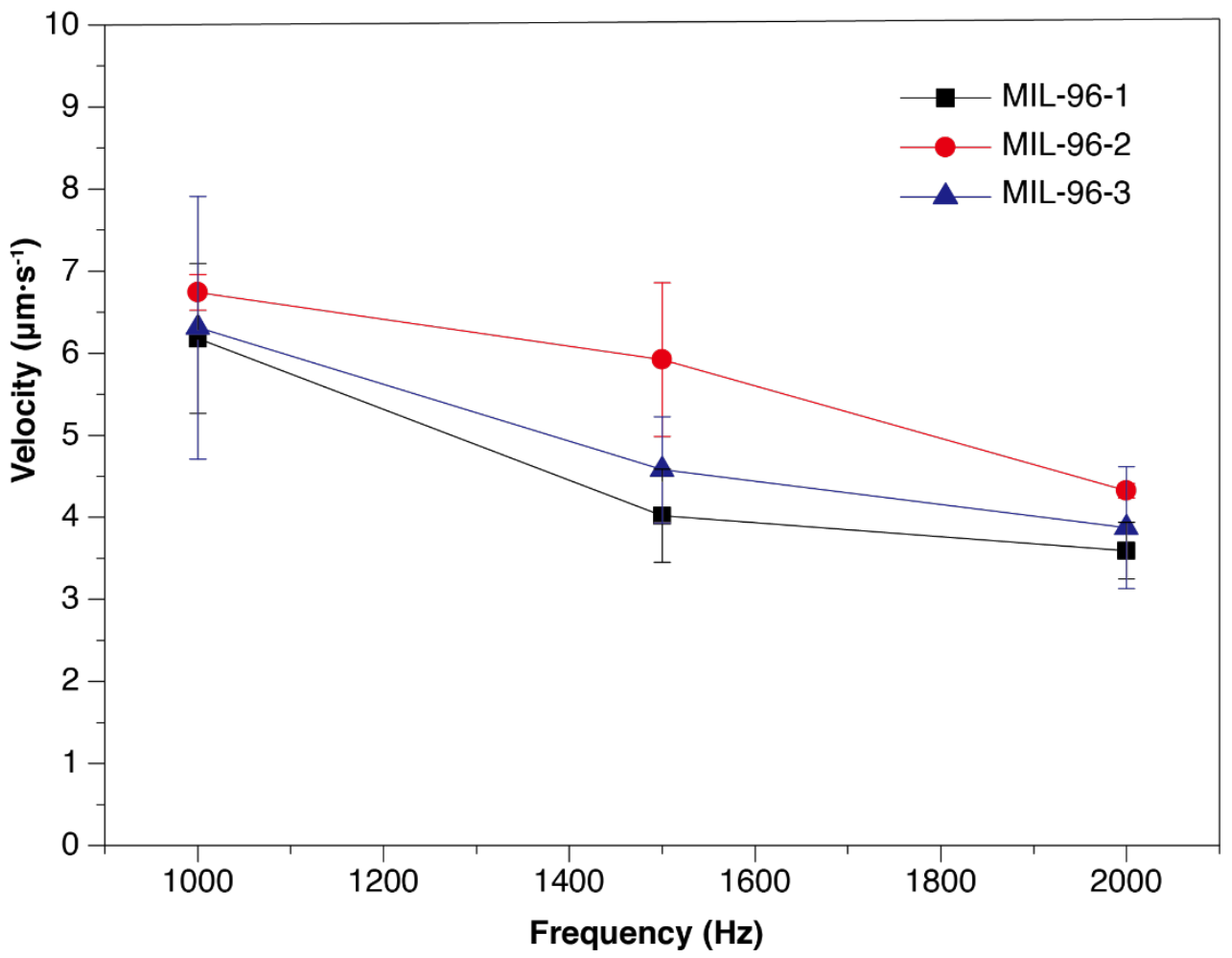

Figure S6. The propulsion velocity of MIL-96 particles with different truncations: velocity (MIL-96-2) > velocity (MIL-96-3) > velocity (MIL-96-1). They differ not only in the degree of truncation but also the size of particle (Table S2). In fact, both the (002) and (101) facets contribute to the propulsion. It is yet challenging to keep one factor constant in the synthesis and precisely derive how the degree of truncation affects the propulsion velocity. 

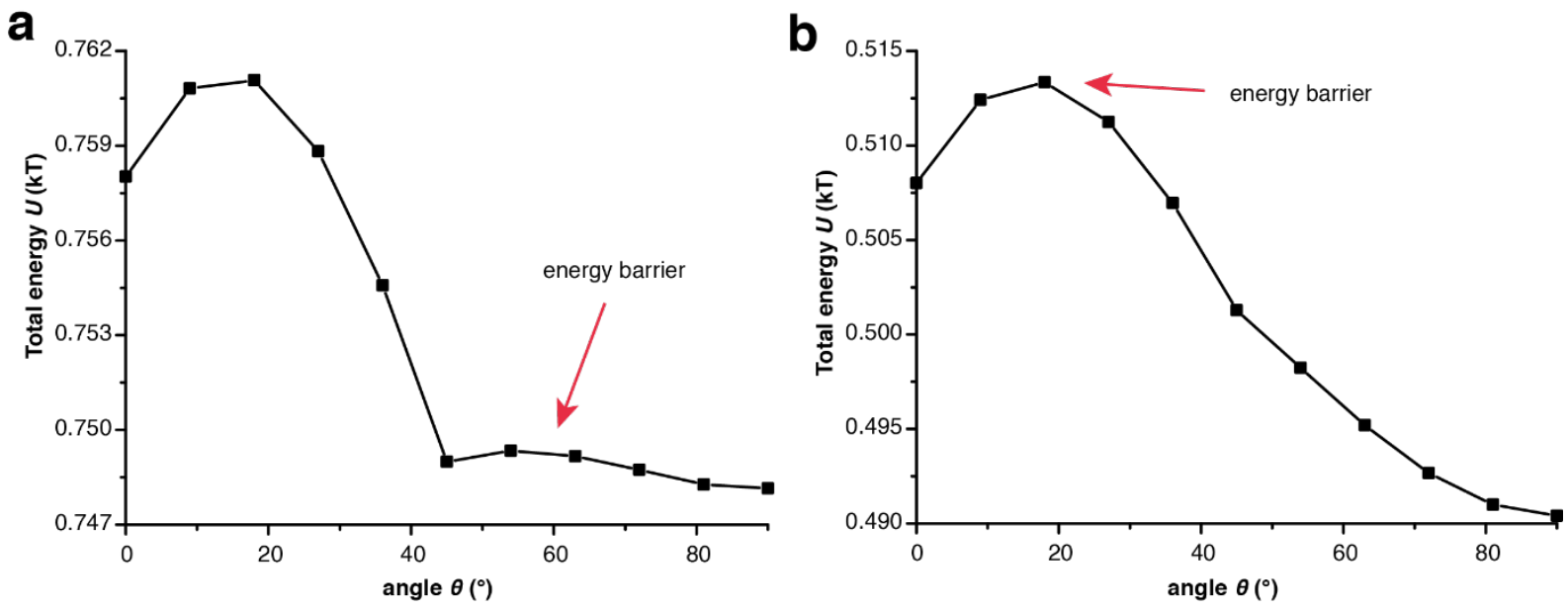

Figure S7. Energy barrier on the theoretical energy profile of MIL-96 particles. Particles may arrest in the active state (a) and steady state (b) when they transition from the steady state to the overexcited state. 


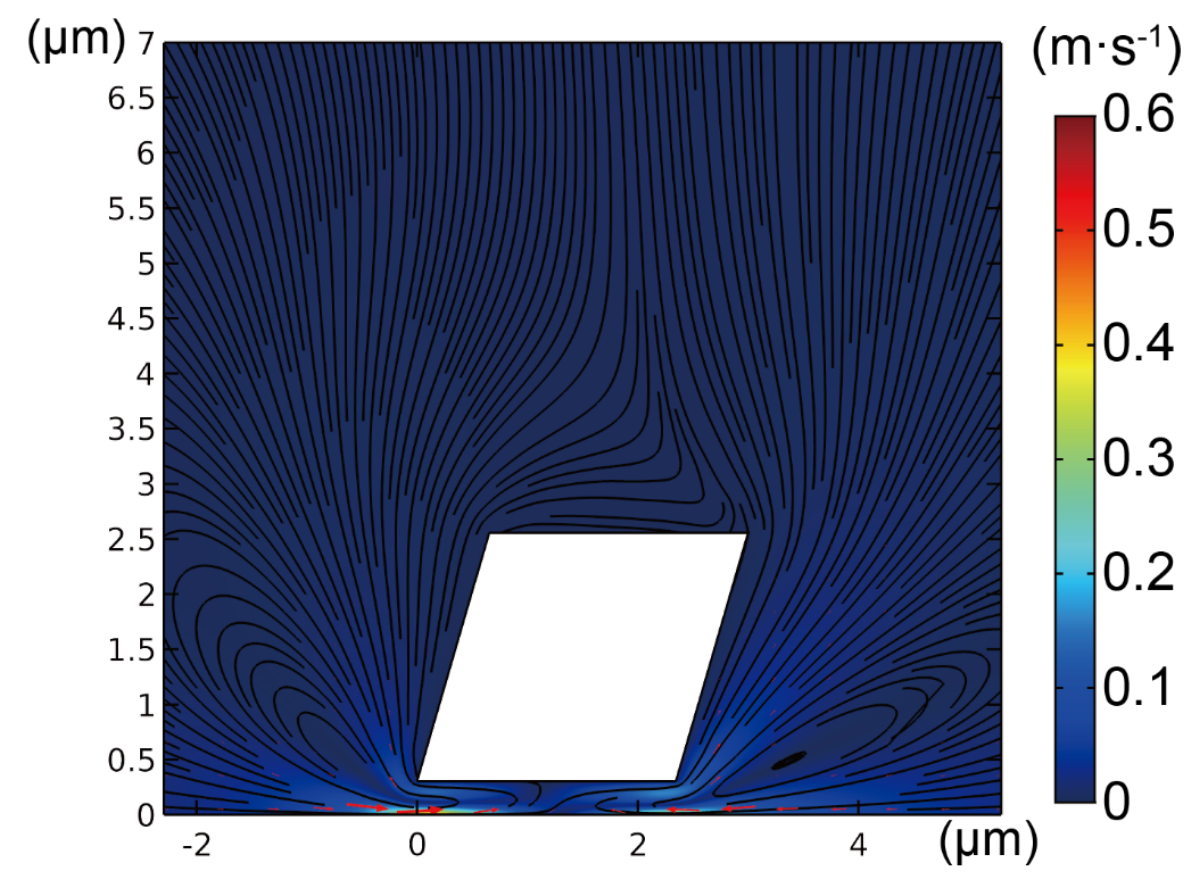

Figure S8. Simulated EHD flow (color map, streamline and small red arrow) around MIL-88B particle.

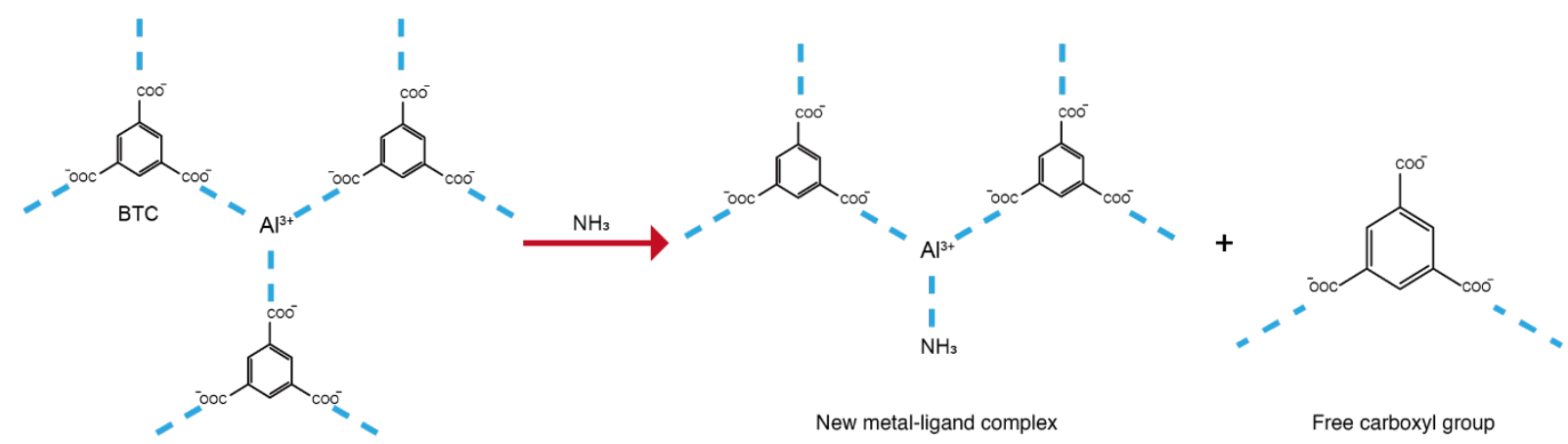

Metal-ligand complex in MIL-96

Figure S9. Ligand exchange leads to changes in surface charges (zeta potentials) for MIL-96 particles. When introducing ammonia $\left(\mathrm{NH}_{3} \bullet \mathrm{H}_{2} \mathrm{O}\right)$, the zeta potentials vary due to ligand exchange instead of changing the $\mathrm{pH}$ values of the system. As a control experiment, zeta potentials of MIL-96 particles in $\mathrm{NaOH}$ or $\mathrm{NH}_{4} \mathrm{Cl}$ solution do not change, suggesting the key role is the $\mathrm{NH}_{3}$. In addition, glycine also affects the zeta potential, indicating that $-\mathrm{NH}_{2}$ group is effective. MOFs are coordination network, which can be easily destroyed by strong ligand ${ }^{6}$, while ammonia of high concentrations indeed destroys the MIL-96 particles. 
Table S3. Zeta potentials of MOF particles after different modification

\begin{tabular}{|c|c|c|c|}
\hline Group & Zeta potential & Group & Zeta potential \\
\hline $\begin{array}{l}\text { MIL96-3 in } 1.48 \\
\text { mM glycine } \\
\text { without sodium } \\
\text { hydroxide }\end{array}$ & $(-4.2 \pm 0.9) \mathrm{mV}$ & $\begin{array}{l}\text { MIL96-3 in } 1.48 \\
\text { mM glycine and } \\
0.74 \text { mM sodium } \\
\text { hydroxide }\end{array}$ & $(-45.0 \pm 4.2) \mathrm{mV}$ \\
\hline $\begin{array}{l}\text { MIL-96-3 in } 1.48 \\
\text { mM glycine and } \\
1.48 \mathrm{mM} \text { sodium } \\
\text { hydroxide }\end{array}$ & $(-62.9 \pm 4.1) \mathrm{mV}$ & $\begin{array}{l}\text { MIL-96-3 in } 250 \\
\text { mM CTAB solution }\end{array}$ & $(+34.7 \pm 1.1) \mathrm{mV}$ \\
\hline MIL-88B & $(+19.8 \pm 0.5) \mathrm{mV}$ & ZIF-8 (cubic) & $(+7.89 \pm 0.99) \mathrm{mV}$ \\
\hline ZIF-8 (RD) & $(-5.59 \pm 1.84) \mathrm{mV}$ & UiO-66 & $(+11.4 \pm 0.4) \mathrm{mV}$ \\
\hline
\end{tabular}



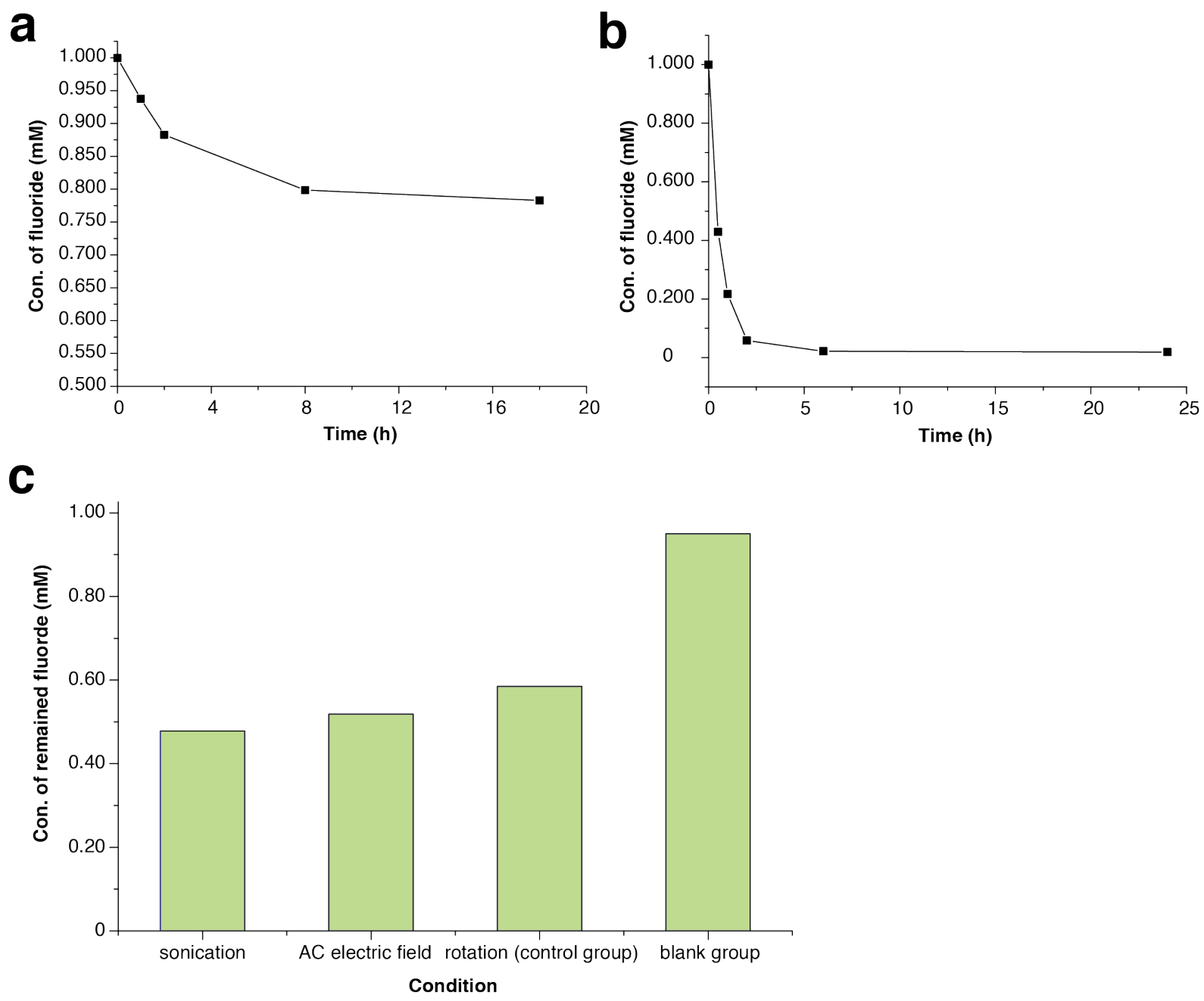

Figure S10. Fluoride removal by MIL-96 particles. (a) $2.0 \mathrm{mg}$ of MIL-96-1 is added into 10 $\mathrm{mL}$ of $0.001 \mathrm{M} \mathrm{NaF}$ aqueous solution in a vial, which was kept under rotating condition. The fluoride concentration was measured at timed intervals using a $\mathrm{pH}$ meter (Leici, PHS-25) with a fluoride ion electrode (Leici, pF-2-01) and a reference electrode (Leici, 232-01). $2.0 \mathrm{mg}$ of MIL-96-1 absorbed in total of $0.041 \mathrm{mg}$ of fluoride. (b) $20 \mathrm{mg}$ of MIL-96-1 was added into 5 $\mathrm{mL}$ of $0.001 \mathrm{M} \mathrm{NaF}$ aqueous solution, and the mixture was kept rotating. $0.095 \mathrm{mg}$ of fluoride were absorbed completely. (c) $8 \mathrm{mg}$ of MIL-96-1 is added into $2 \mathrm{~mL}$ of $0.001 \mathrm{M} \mathrm{NaF}$ aqueous solution, which is divided into four group with different conditions (sonication, AC electric field $\left(f=1 \mathrm{kHz}, V_{\mathrm{pp}}=15 \mathrm{~V}\right.$, a larger electric cell with a height of $1 \mathrm{~mm}$ is used), rotation (control group), and steady (blank group)) for 30 mins before remaining fluoride in solution was measured. The group under AC electric perform better than the control group in terms of removing fluoride. 


\section{Supplementary Discussions}

Supplementary Discussion 1. Determining the orientation of MOF particles.

The behaviors of MOF particles are observed under an inverted microscope. The microscope focus is adjusted to near the bottom substrate, where particles settle due to gravity. We use the Trackpy (Python package) to track the particles location. The particle tracking algorithm (PTA) is to find group of pixels with certain grey scale values, which represents the particles (or part of a particle). By analyzing sequences of pictures, the trajectory of particle propulsion can be obtained. To determine the orientation of MOF particles, the shape/symmetry of the particles are considered together with particle tracking (if needed). The analysis is shown below, which depends on specific MOF type/shape.

Orientation of MIL-96 particles. MIL-96 particles adopt the truncated hexagonal bipyramidal shapes (THBP) and have two hexagonal (002) facets and twelve trapezoidal (101) facets. For the particles at the steady state and overexcited state, it is easy to distinguish the orientation from microscope images and videos (Figure S11 and Supporting Information Video 1). The former appears as a regular hexagon, while the latter looks like an ellipsoid (Figure S11).

a

Steady state

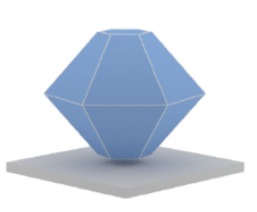

b

Overexcited state

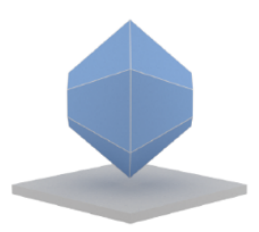

Cartoon
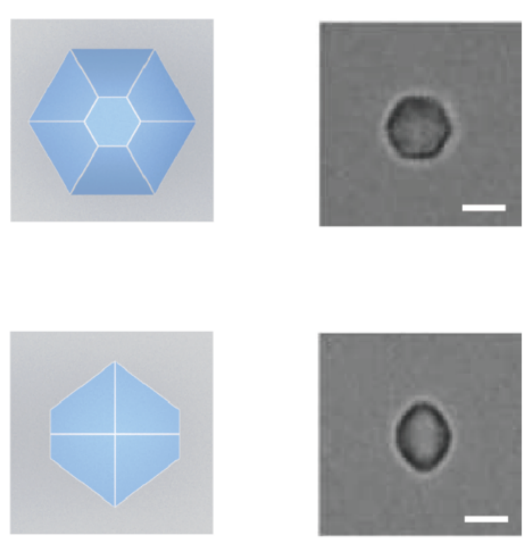

Top view

Microscope image

Figure S11. MIL-96 particles adopting the steady (a) and overexcited state (b). The relevant cartoons and microscope images are shown. Scale bar: $2 \mu \mathrm{m}$. 
For particles at the active state, their orientation is determined by the particle's outline (contrast of microscope images). As shown in Figure S12a, when the particle propels, the image of the particle features three lines with different darkness $\left(\mathrm{L}_{1}\right.$, dark; $\mathrm{L}_{2}$ and $\mathrm{L}_{3}$, light). They separate the particles into two regions, $\mathrm{A}$ and $\mathrm{B}$, and the area of $\mathrm{A}$ is greater than that of $\mathrm{B}$. By comparing to the cartoon model, $\mathrm{L}_{2}$ in the middle should be the bottom edge of the trapezoidal (101) facet in contact with the substrate. Region A should therefore be the image that combines the (002) facet and (101) facet close to the substrate; region B should be the image of the (101) facet near the bottom. These assignments match the cartoon and verify the orientation of MIL96 as they propel, where the bottom (002) facet is pointing to the front.

a

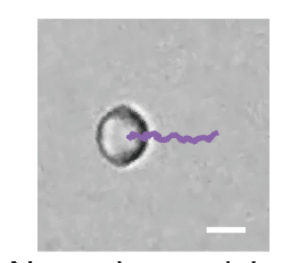

Normal propulsion
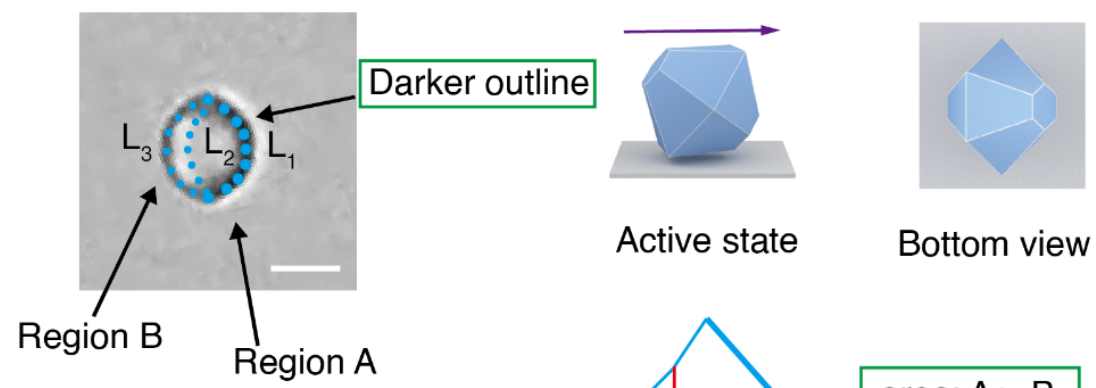

Active state

Bottom view

area: $\mathrm{A}>\mathrm{B}$

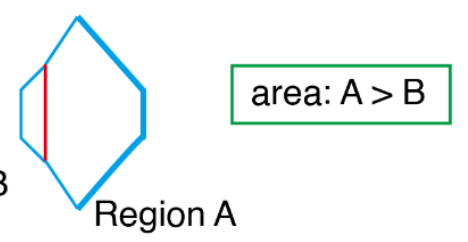

Cartoon of Microscope view

b

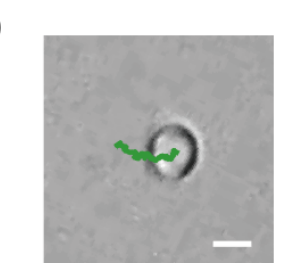

Reversed propulsion

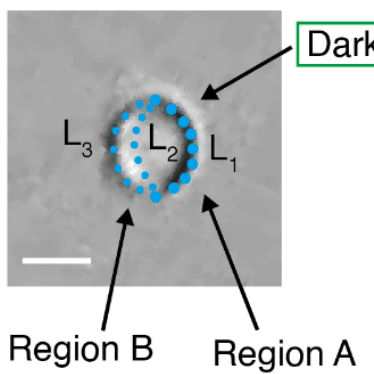

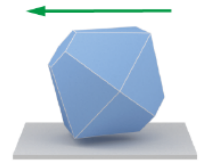

Active state

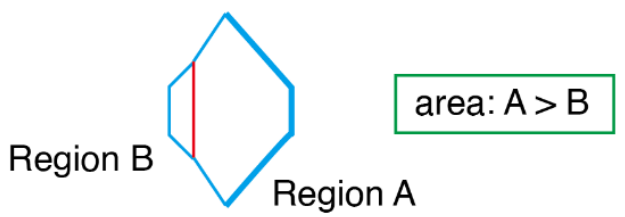

Cartoon of Microscope view

Figure S12. Propulsion of MIL-96 particles (a) at a low zeta potential and reversed propulsion (b) at a high zeta potential of propulsion. Both adopt the active state and their orientation can be determined by contrast features in optical images as compared to cartoon models. Scale bar: $2 \mu \mathrm{m}$. 
Similarly, when the particles with a high zeta potential $|\zeta|$ propel, similar lines and regions can be observed. However, propulsion direction is opposite to the arrangement of region A and B (Figure S12b).

Orientation of MIL-88B particles. MIL-88B particles feature a hexagonal bipyramidal shape (HBP) and have twelve identical triangular (101) facets. At active state, they sit by one of its (101) facets. Under inverted microscope, three vertexes of the bottom triangle are observed, confirming that MIL-88B particle propels toward the hexagonal tips that is close to the substrate (Figure S13a). At the overexcited state, a hexagram star-like pattern can be clearly observed, confirming that they are aligned with the main axis parallel to the electric field (Figure S13b).

a

\section{Propulsion}

Active state
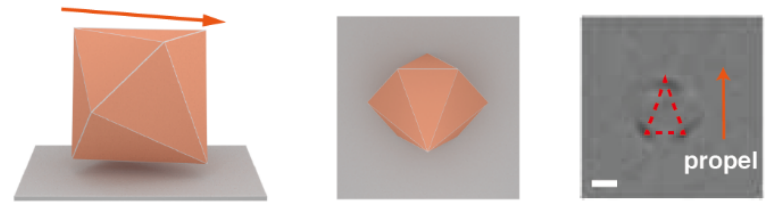

b

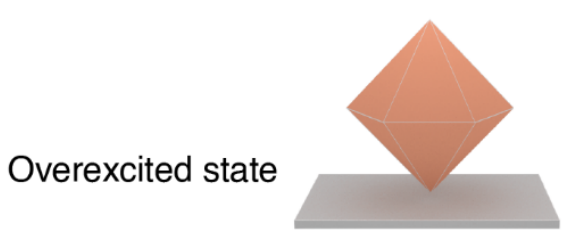

Cartoon

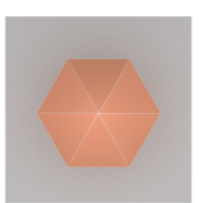

Top view Microscope image

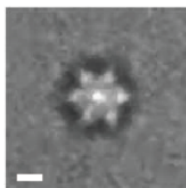

Figure S13. MIL88B particles adopting the active state (a) and the overexcited state (b). Scale bar: $1 \mu \mathrm{m}$.

Orientation of ZIF-8 particles. Cubic ZIF-8 particles appear as squares which can be easily distinguished. The rhombic dodecahedral (RD) ZIF-8 particle appears as hexagonal-like shapes. In both cases, the particles do not propel under AC electric field.

Orientation of UiO-66 particles. UiO-66 particles are octahedral in shape with eight identical regular triangular facets. At the overexcited state, their main axis is aligned with the electric field. The microscope image features a square shape with four internal boundaries (Figure S14). 


\section{Overexcited state}

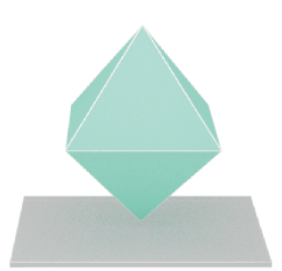

Cartoon

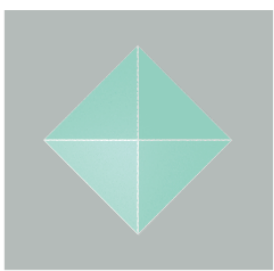

Top view

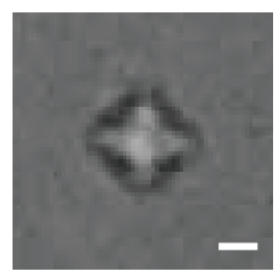

Microscope image

Figure S14. UiO-66 particles adopt overexcited state. Scale bar: $1 \mu \mathrm{m}$.

At the active state, when the particle sits by one triangular facet on the substrate, three dark spots of bottom triangle (A, B, and C) and three blurred spots of top triangle (D, E, and F) can be observed (Figure S15a). When the particle tilts to propel, one of three bottom vertices is away from the substrate. As a result, only two dark spots are observed (Figure S15b). The missing dark spot, $\mathrm{B}$, is in the opposition direction to the propulsion, confirming the particle propel with the upper vertex forward. Each dark spot can be tracked by particle tracking algorithm (PTA) (Figure S15). Thus, the orientation of particles can be identified by counting the number of dark spots during the particles propel, turn, or roll (as shown in Figure 7 in the main text).

a

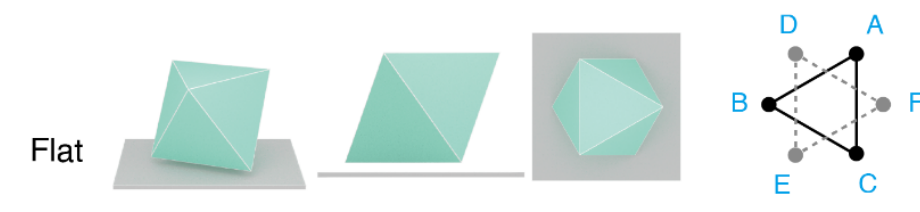

b
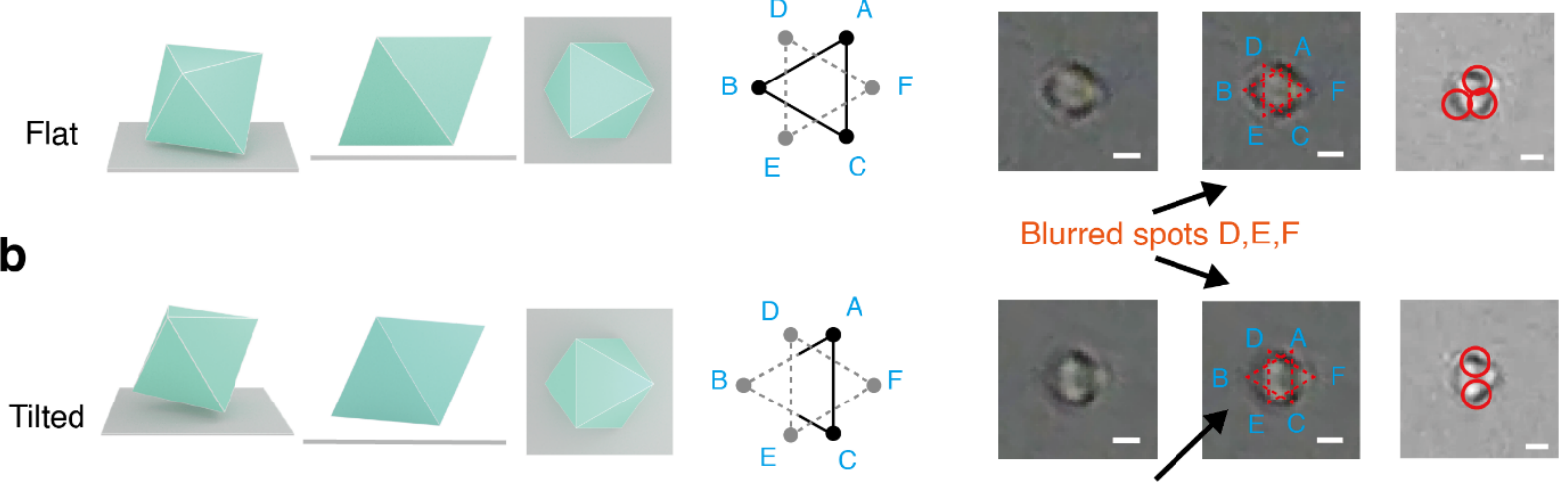

Blurred spot B when tilted Cartoon Side view Top view $\begin{array}{r}\text { Bottom facet "A-B-C" } \\ \text { and top facet "D-E-F" }\end{array}$

Microscope image

PTA image

Figure S15. UiO-66 adopting active state (a), which tilts slightly when propelling (b). The cartoons and microscope images are shown. Scale bar: $1 \mu \mathrm{m}$. 
Supplementary Discussion 2. Finite-element simulation of the relation between the dihedral angle and resulting lateral EHD profile.

The relation between the dihedral angle $\alpha$ and resulting lateral EHD profile is verified by studying a half of hypothetic hexagons. As shown in Figure S16, when the dihedral angle increases, the nonuniform electric field around the side face gets stronger, indicating that the higher inclination of side facet leads to more distortion of the nearby electric field. As a result, the EHD flow is attenuated.

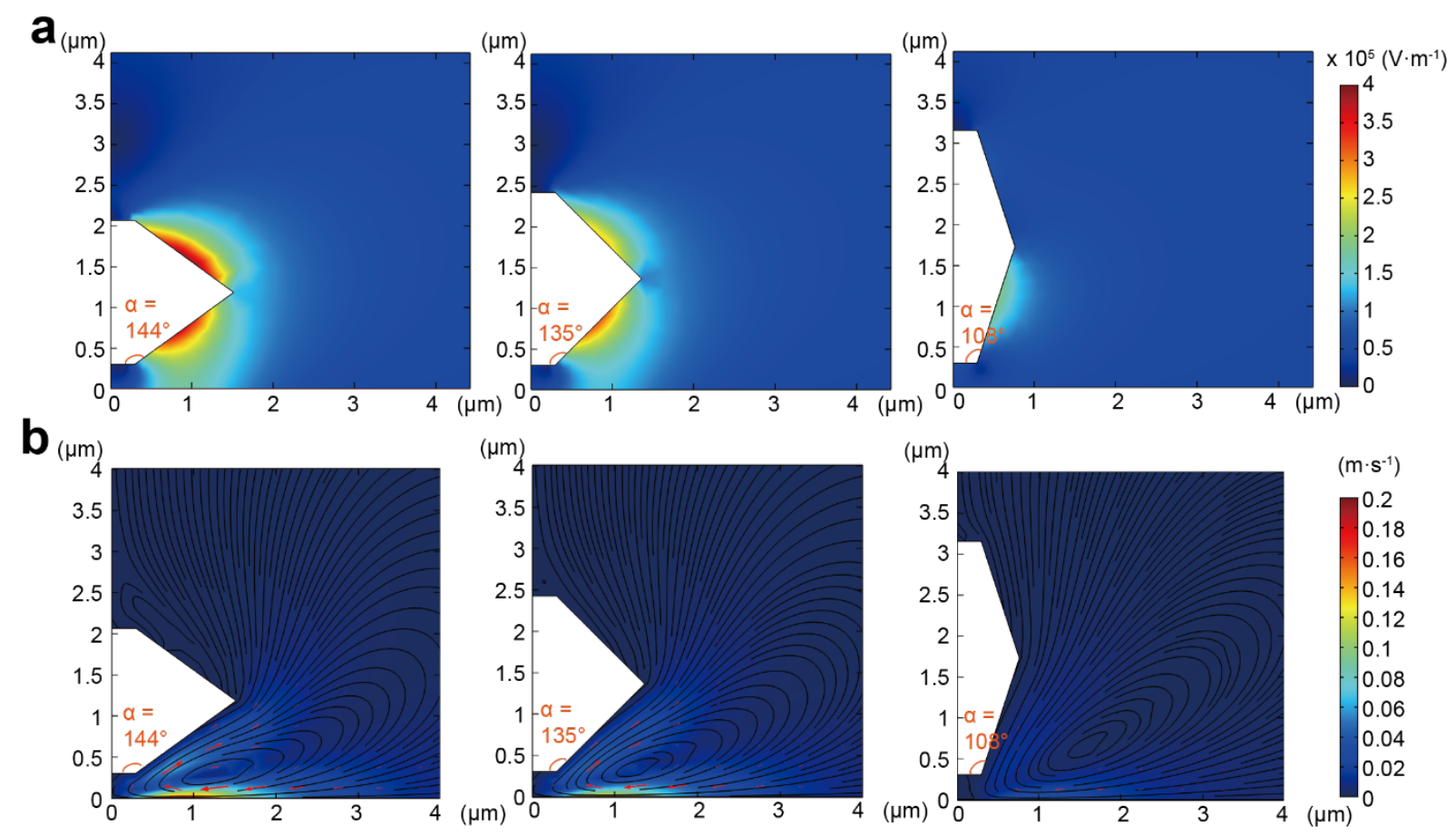

Figure S16. Simulated electric field strength distribution (a, color map) and flow (b, color map, streamline and red arrow) around hypothetic polyhedral particle with different dihedral angle $\alpha$. 
Supplementary Discussion 3. The theoretical model for particle orientation

We model MIL-96 particles by placing Lorentz atoms on a cubic lattice in $x$-z plane with $a$ $=100 \mathrm{~nm}$ as the size of unit cell. Considering the angle between the (002) facet and the (101) facet is around $134^{\circ}$, close to $135^{\circ}$, the way of cubic stacking is suitable for building MIL-96 particles. It is a semi-2D model in $x-z$ plane since we only build one layer of Lorentz atoms in $y$ direction to simplify the calculation, which is the cross-section of MOF shown in the Figure 4. Radius of Lorentz atom is $(\sqrt{ } 2)^{*} a / 4$. Number density of Lorentz atom, $n$ is calculated by $4 / \mathrm{a}^{3}$. The distance between particles' lowest point to the electrode, $h$ is $100 \mathrm{~nm}$. The electric field strength is initially set as $5 * 10^{4} \mathrm{~V} \cdot \mathrm{m}^{-1}$. Although MOF particles are composed of the framework and medium (in this case water), which yet are distributed orderly and uniformly, the whole particles can be regarded as homogenous material so that each Lorentz atom has same physical properties including polarizability $\alpha_{0}$, mass density, and the dielectric constant. Mass density of Lorentz atom is regarded as $1200 \mathrm{~kg} \cdot \mathrm{m}^{-3}$ referring to a calculated data. The relative dielectric constant of medium and particles is 78 and 3, respectively. For MIL-96, the equatorial width is fixed as 15 Lorentz atoms length. The truncation is changed by set different height along the $z$ direction. We label the MIL-96 particle as MIL-96-S- $i(i=\mathbf{1 , 2 , 3 , 4 , 5})$, shown in Table S4 and Figure S17a. More geometry parameters are shown in Table S4.

For UiO-66, cubic lattice is deformed by shifting each line to reach an inclination angle same to octahedron, $70.5^{\circ}$ (Table S5, Figure S17b, Figure S18).

Next, we show the calculation of electric potential energy. The dipole moment follows:

$$
\boldsymbol{p}_{i}=\alpha_{0} \boldsymbol{E}_{i}
$$

$\alpha_{0}$ is calculated by the Clausius-Mossotti relation:

$$
\frac{\alpha_{0} n}{\varepsilon_{\mathrm{m}}}=\frac{3}{4 \pi}\left(\frac{{ }^{\varepsilon_{\mathrm{p}}} / \varepsilon_{\mathrm{m}}-1}{\varepsilon_{\mathrm{p}} / \varepsilon_{\mathrm{m}}+2}\right)
$$

Where, $n$ is number density of Lorentz atoms and $\varepsilon_{\mathrm{m}}$ and $\varepsilon_{\mathrm{p}}$ are the dielectric constant of the medium and particles, respectively. $\boldsymbol{E}_{i}$ is the local electric field experienced by the atom $i$ :

$$
\boldsymbol{E}_{i}=\boldsymbol{E}_{0}+\frac{1}{\varepsilon_{\mathrm{m}}} \sum_{j=1}^{N} \boldsymbol{T}_{i j} \cdot \boldsymbol{p}_{j}
$$


where $\boldsymbol{T}_{i j}$ is the dipole-dipole tensor:

$$
\boldsymbol{T}_{i j}= \begin{cases}\frac{3 r_{i j} r_{i j}}{\left|r_{i j}\right|^{2}}-\boldsymbol{I} & \text { if } i \neq j \\ 0, & \text { if } i=j\end{cases}
$$

where $\boldsymbol{r}_{i j}=\boldsymbol{r}_{i}-\boldsymbol{r}_{j}$. Finally, we get a set of matrix equations:

$$
\boldsymbol{p}_{i}-\frac{\alpha_{0}}{\varepsilon_{m}} \sum_{j=1}^{N} \boldsymbol{T}_{i j} \cdot \boldsymbol{p}_{j}=\alpha_{0} \boldsymbol{E}_{0}
$$

By solving them, the dipole moment of each Lorentz atom and thus the total electric energy in Eq. 3 can be obtained.

Table S4. Geometry parameters of hypothetic MIL-96 particles in theoretical model.

\begin{tabular}{|c|c|c|c|c|c|}
\hline MIL-96 & S-1 & S-2 & S-3 & S-4 & S-5 \\
\hline Equatorial width/ $\mu \mathrm{m}$ & 1.4 & 1.4 & 1.4 & 1.4 & 1.4 \\
\hline Half height/ $\mu \mathrm{m}$ & 0.6 & 0.5 & 0.4 & 0.3 & 0.2 \\
\hline Bottom $/ \mu \mathrm{m}$ & 0.2 & 0.4 & 0.6 & 0.8 & 1 \\
\hline Degree of truncation & 0.14 & 0.29 & 0.43 & 0.57 & 0.71 \\
\hline
\end{tabular}

Table S5. Geometry parameter of UiO-66 particles in theoretical model.

\begin{tabular}{|c|c|c|}
\hline & Side $/ \mu \mathrm{m}$ & Bottom $/ \mu \mathrm{m}$ \\
\hline UiO66 & 0.8 & 0.8 \\
\hline
\end{tabular}


a

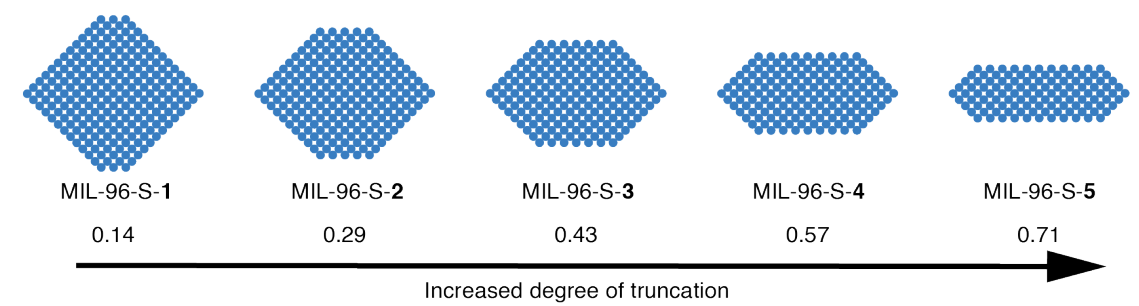

b

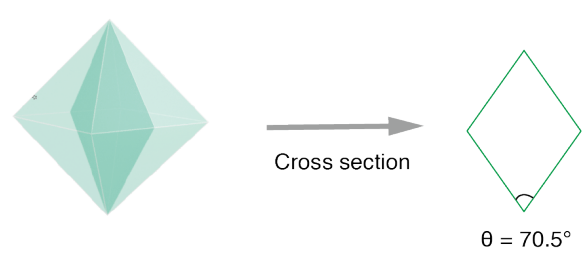

UiO-66

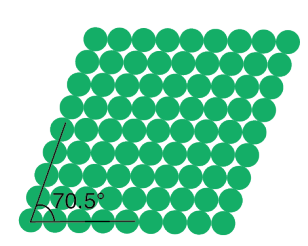

CDM model

Figure S17. Theoretical geometry model of MIL-96 with different truncation (a) and UiO-66 (b).

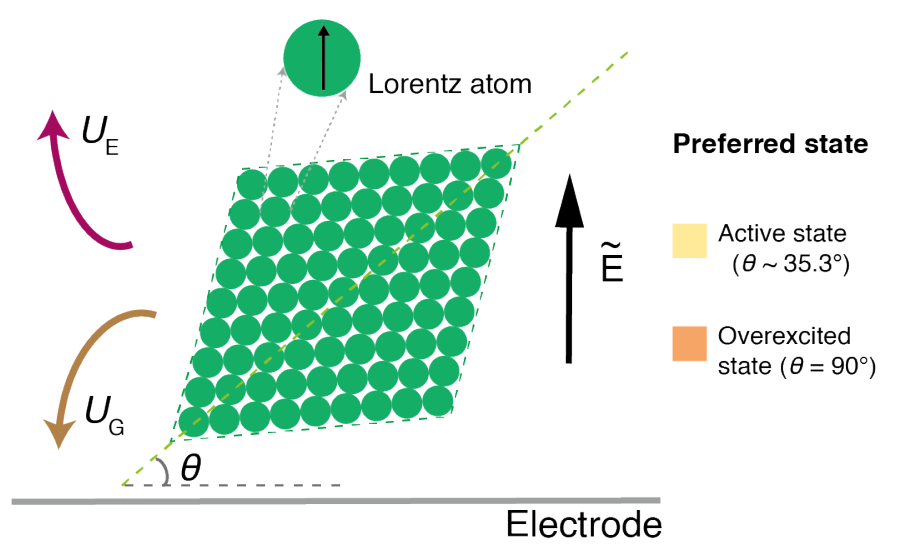

Figure S18. Schematic theoretical model regarding the orientation of the UiO-66 particle. 


\section{Video Descriptions}

Video 1: MIL-96 THBP particle adopts different orientations according to the strength of AC electric field. When the field is turned on at frequency $f=1 \mathrm{kHz}$, voltage $V_{\mathrm{pp}}=8 \mathrm{~V}$, the particle transitions from the steady state to the active state. When the field is turn on at $f=1 \mathrm{kHz}, V_{\mathrm{pp}}=$ $10 \mathrm{~V}$, the particle transitions from the steady state to the overexcited state.

Video 2: Propulsion of MIL-96 THBP particles under an AC electric field ( $f=1 \mathrm{kHz}$, voltage $=8 \mathrm{~V})$. The particles adopt the active state.

Video 3: The delayed switch between different orientations indicates the presence of an energy barrier. The MIL-96-1 particle stays temporarily at the active state during the transition from the steady state to the overexcited state. The MIL-96-5 particles exhibit an inertia of the steady state during the transition to the overexcited state.

Video 4: MIL-88B HBP particle propels under an AC electric field $\left(f=1 \mathrm{kHz}, V_{\mathrm{pp}}=8 \mathrm{~V}\right)$.

Video 5: ZIF-8 particles (rhombic dodecahedral and cubic) show no propulsion under an AC electric field.

Video 6: The octahedral UiO-66 particle propels and performs complex motions under an AC electric field. The particle changes its propulsion head among the three top vertices and then turns. The particle propels by rolling continuously $\left(f=800 \mathrm{~Hz}, V_{\mathrm{pp}}=7.5 \mathrm{~V}\right)$.

Video 7: MIL-96 particle with a zeta potential $\zeta=-87.23 \mathrm{mV}$ propels in a reversed direction compared to the previous case, where the particles have lower zeta potentials $(-29.07 \mathrm{mV} \sim$ $63.47 \mathrm{mV})$. 


\section{Supplementary References}

1. Sindoro, M.; Jee, A.-Y.; Granick, S., Shape-selected colloidal MOF crystals for aqueous use. Chem. Commun. 2013, 49 (83), 9576.

2. Wang, S.; McGuirk, C. M.; Ross, M. B.; Wang, S.; Chen, P.; Xing, H.; Liu, Y.; Mirkin, C. A., General and Direct Method for Preparing Oligonucleotide-Functionalized Metal-Organic Framework Nanoparticles. J. Am. Chem. Soc. 2017, 139 (29), 9827-9830.

3. Pan, Y.; Heryadi, D.; Zhou, F.; Zhao, L.; Lestari, G.; Su, H.; Lai, Z., Tuning the crystal morphology and size of zeolitic imidazolate framework- 8 in aqueous solution by surfactants. CrystEngComm 2011, 13 (23), 6937-6940.

4. Cai, X.; Lin, J.; Pang, M., Facile Synthesis of Highly Uniform Fe-MIL-88B Particles. Cryst. Growth Des. 2016, 16 (7), 3565-3568.

5. Ristenpart, W. D.; Aksay, I. A.; Saville, D. A., Electrohydrodynamic flow around a colloidal particle near an electrode with an oscillating potential. J. Fluid Mech. 2007, 575, 83109.

6. Yu, D.; Shao, Q.; Song, Q.; Cui, J.; Zhang, Y.; Wu, B.; Ge, L.; Wang, Y.; Zhang, Y.; Qin, Y.; Vajtai, R.; Ajayan, P. M.; Wang, H.; Xu, T.; Wu, Y., A solventassisted ligand exchange approach enables metal-organic frameworks with diverse and complex architectures. Nat. Commun. 2020, 11 (1), 1-10. 